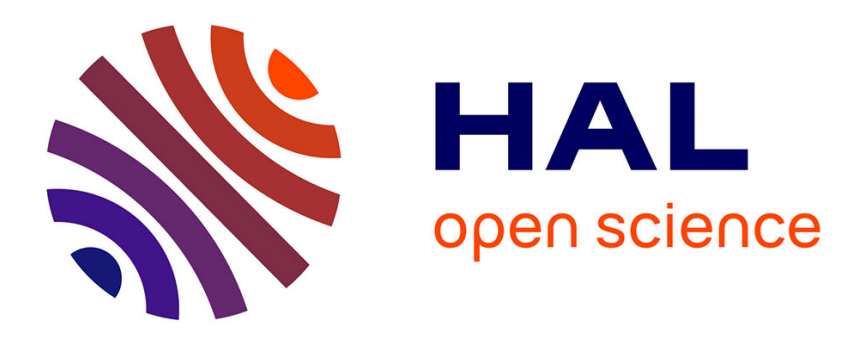

\title{
De la ville réelle à la ville rêvée: Espaces urbains dans l'Espagne de la fin du XVIIIe siècle
}

\author{
Marc Marti
}

\section{To cite this version:}

Marc Marti. De la ville réelle à la ville rêvée: Espaces urbains dans l'Espagne de la fin du XVIIIe siècle. Paysages urbains, XVIe-XXe siècle, Dec 1998, Grasse, France. pp.87-100. halshs-00568043

\section{HAL Id: halshs-00568043 \\ https://shs.hal.science/halshs-00568043}

Submitted on 25 Feb 2011

HAL is a multi-disciplinary open access archive for the deposit and dissemination of scientific research documents, whether they are published or not. The documents may come from teaching and research institutions in France or abroad, or from public or private research centers.
L'archive ouverte pluridisciplinaire HAL, est destinée au dépôt et à la diffusion de documents scientifiques de niveau recherche, publiés ou non, émanant des établissements d'enseignement et de recherche français ou étrangers, des laboratoires publics ou privés. 


\section{De la ville réelle à la ville rêvée: Espaces urbains dans l'Espagne de la fin du XVIIle siècle}

Marc MARTI, Université de Nice, CNA, Département d'espagnol

Il y a maintenant bon nombre d'années que l'espace, en tant que domaine de recherche, n'est plus un domaine réservé à la géographie traditionnelle. En 1982, le géographe Robert Ferras dressait déjà un bilan prometteur des autres approches possibles autour de la notion d'espace vécu1. La diversité des intervenants à ce colloque est là pour démontrer qu'il ne s'était pas trompé. Dans le cadre de cette communication, nous voudrions essayer d'analyser les rapports entre l'espace urbain réel de l'Espagne de la fin du XVIIIe et ses représentations dans les textes économiques. Nous suivrons, pour ce faire, les suggestions de Robert Ferras, soit «trois angles d'attaque: la pratique d'un support objectif que l'on peut décrire, sa représentation, qui n'est que subjective, l'imaginaire qui relève de l'idéologie2»».

Le XVIIIe siècle fut, en Espagne comme en Europe, une période de mutation objective de l'espace urbain, mais la péninsule possède ses propres spécificités. L'essor démographique contribua sans aucun doute au phénomène. Nous essaierons de déterminer dans quelle mesure l'augmentation de la population a conditionné ce changement.

L'espace urbain va se transformer de façon visible, comme en témoignent différentes réalisations architecturales, toujours présentes à notre époque. En parallèle, les gouvernants appliqueront des réformes visant à administrer la ville d'une autre façon. Mais ces nouveautés, à quelles intentions et à quelles nécessités correspondaient-elles?

Par ailleurs, les traités économiques donnent une image très souvent négative de l'espace urbain. La ville est accusée de tous les maux, et la plupart des analyses lui sont défavorables. Il y a là une évidente contradiction entre les données objectives, qui montrent que la ville est choyée par les gouvernants, et celles, subjectives, des

${ }^{1}$ Robert Ferras, «"L'espace vécu": une direction de recherche en géographie», Imprévue $\mathrm{n}^{\circ} 2$, Montpellier, CERS, 1982, p. 17-27.

${ }^{2}$ Robert Ferras, art. cit., p. 25. 
économistes. C'est cette contradiction que nous essaierons d'expliquer par l'analyse de l'imaginaire et de l'idéologie des élites de cette Espagne de la fin du XVIIIe.

\section{La croissance urbaine}

\subsection{Un essor démographique}

Pour l'Espagne, le XVIIIe siècle est marqué par une croissance démographique notable. Les facteurs expliquant cet essor sont très nombreux mais sont pratiquement les mêmes que dans le reste de l'Europe: la fin des guerres meurtrières, la quasi-disparition de la peste et la reprise économique généralisée. Du point de vue biologique, c'est encore, comme aux époques précédentes, la hausse des naissances, et non la baisse de la mortalité, qui est à l'origine du phénomène. Le taux de fécondité, étroitement lié au taux de nuptialité, reste très sensible aux facteurs conjoncturels ${ }^{3}$. La mortalité ne varie que très peu et de façon beaucoup moins élastique. La population s'accroît naturellement, avant les progrès de la médecine et de l'hygiène, qu'il vaudrait mieux situer au XIXe siècle, et encore, dans sa deuxième partie. C'est toujours à un régime démographique d'ancien type auquel nous avons affaire, régime qui devient excédentaire, grâce à l'amélioration des conditions économiques, et ce, malgré les hausses brusques des décès enregistrées localement, dues la plupart du temps à des mauvaises récoltes engendrant des disettes ou à des fièvres endémiques, fréquentes dans le sud de la péninsule.

A la différence d'autres pays européens, de nombreux recensements furent réalisés pendant ce siècle, démontrant la relative modernité de l'administration espagnole et sa sensibilité aux problèmes de population. Les données brutes sont inexactes mais permettent d'avoir un ordre de grandeur acceptable sur le nombre d'habitants. En données corrigées, l'Espagne serait passée de 7,5 millions d'habitants en 1700 à 9,8 millions à la moitié du siècle pour atteindre les 12 millions en $1808^{4}$.

3Vicente Pérez Moreda, Las crisis de mortalidad en la España interior. Siglos XVI-XIX, Madrid, 1981, p. 53.

${ }^{4}$ Marc Marti, Ville et campagne dans l'Espagne des Lumières (1746-1808), Saint Étienne, 1997, p. 21 sq. 
En effet, la répartition spatiale de cette croissance fut loin d'être homogène. Elle fut beaucoup plus importante dans les zones périphériques (Levant, Galice, Nord-ouest). C'est aussi à partir de cette période qu'allait émerger un nouvel équilibre démographique national. Pour la première fois, la périphérie allait prendre irrémédiablement le dessus sur le centre. Ce basculement s'explique autant par la croissance biologique que par les migrations internes. Les périphéries, dont Barcelone devenait l'éclatant exemple de réussite, allaient drainer de plus en plus d'hommes vers elles ${ }^{5}$.

\subsection{L'essor urbain et ses caractéristiques}

Tout comme les zones côtières, la majorité des villes va connaître un accroissement plus ou moins important mais réel, bien que les tailles atteintes par certaines puissent aujourd'hui nous sembler bien modestes. En effet, seulement une quarantaine de villes comptaient plus de 10000 habitants en 1787. Madrid, avec ses 200000 habitants semblait une monstrueuse capitale aux yeux des contemporains. Barcelone dépassait les 100000 habitants, la population ayant presque triplé depuis 1714. Séville et Valence approchaient aussi la centaine de milliers d'habitants. Les zones les plus urbanisées — pour des raisons historiques et économiques- étaient le Centre, le Sud et le Levant. Sur l'ensemble du territoire, environ $20 \%$ de la population vivait en ville. Malgré sa modestie donc, l'accroissement urbain fut une réalité incontournable et perceptible, possédant ses propres caractéristiques.

Dans l'espace urbain, les naissances sont, pour des raisons évidentes - manque de place, taux plus élevé de célibataires-, bien moins importantes qu'à la campagne. En fait, toutes les études montrent que la population urbaine doit sa croissance, pour une grande part, à l'arrivée régulière d'immigrants ruraux $^{6}$. Ce flux migratoire peut, en gros, être divisé en deux types:

-Une immigration régulière, qui dépend presque directement des possibilités de la ville. Les immigrants venaient tenter leur chance en ville. Les salaires, plus élevés qu'en province, exerçaient un attrait non

5Pierre Vilar, La Catalogne dans l'Espagne Moderne, SEVPEN, 1962, p. 62 sq.

6Jacques Soubeyroux, Paupérisme et rapports sociaux à Madrid au XVIIIe siècle, Lille, Atelier de reproduction des thèses universitaires de Lille III, 1978, p. 20 sq. 
négligeable. Par exemple, les différences entre ceux de Léon et de Madrid allaient du simple au quadruple.

-Une immigration irrégulière qui se développait en période de disette. Les pauvres affluaient alors vers la ville, espérant y trouver un travail ou, au moins, de quoi subsister en mendiant ou en profitant de la soupe populaire. La fin du siècle, à cause de mauvaises récoltes et de l'aggravation de la conjoncture économique, verra augmenter fortement cette errance de crise. La ville n'était donc pas une «fabrique de pauvres》» mais plutôt un "aimant». C'est à la campagne que revenait le triste rôle de créer la majorité des pauvres.

Telles sont donc, schématiquement, les caractéristiques de l'essor démographique des villes au XVIIIe, un essor qui va entraîner une modification notable de l'espace urbain.

\section{Les principales mutations de l'espace urbain}

\subsection{Les modifications architecturales}

Nous ne pouvons ici rendre compte de la situation de toutes les villes, mais nous essaierons de donner quelques exemples qui nous semblent significatifs.

A Madrid, on observe un accroissement des quartiers périphériques, où la densité de la population augmente de 50\% à 100\% entre 1717 et 1769 . Ce phénomène s'explique avant tout par le fait qu'il n'y a pas de faubourgs et que le principal pôle d'attraction était la domesticité, une activité forcément située au centre de la ville, dans les plus riches demeures. A Barcelone, la croissance urbaine modifiera bien plus le paysage. En effet, les villages environnants, entre 1718 et 1787, voient leur population tripler, voire quadrupler. L'activité marchande et portuaire de la capitale catalane dynamise sa périphérie, qui se spécialise alors dans la production manufacturière ${ }^{8}$.

${ }^{7}$ C'est l'expression employée par Anne-Marie Piuy, Villes et Campagnes $X V$ XXe siècle, Centre d'Histoire économique et sociale de la région lyonnaise, Lyon, Presses Universitaires, 1977, p. 21: «[...] la ville ancienne est une grande fabrique de pauvres». Nous pensons qu'il convient de la nuancer, car en Espagne, ce sont avant tout les structures agraires qui conditionnent le paupérisme, bien que les salaires urbains fabriquent aussi une classe de pauvres en puissance, comme l'ont démontré les études de Jacques Soubeyroux ( $O p$. cit.).

8Pierre Vilar, Op. cit., pp. 62-63. 
Hormis ces modifications presque «naturelles» et dues à l'essor démographique, on peut noter, en parallèle, des changements imposés par les édiles ou les gouvernants. Madrid, capitale administrative et siège du pouvoir sera bien sûr privilégiée. Une partie des lieux aujourd'hui célèbres de la capitale espagnole sont nés au XVIIIe: promenade du Prado, fontaine de la Cibeles, Puerta de Álcala. Le but, pour les souverains, était de transformer cette petite ville de Castille en une capitale européenne digne de ce nom, capable d'impressionner favorablement les voyageurs, dont le trajet se déroulait le plus souvent d'est en ouest. Le long de cette traversée "touristique" se situaient tous les édifices prestigieux ${ }^{9}$. Jacques Soubeyroux parle de la "construction d'un décor monumental, fait pour le voyageur, qui privilégie quelques secteurs de la ville au détriment de l'ensemble, et qui traduit un traitement théâtral du paysage urbain ${ }^{10}$ ». Le soin apporté à des lieux stratégiques, tels que les entrées et les promenades, s'explique ainsi. L'entrée était la première image que percevait de la ville l'étranger arrivant par la route, au rythme lent de la voiture tirée par des mules. La promenade était ensuite un des espaces privilégiés de la sociabilité urbaine. Elle correspondait à un exercice quotidien codifié, qui existait aussi en France. Cette pratique était l'apanage des classes élevées, qui pouvaient faire étalage de leur richesse et, symboliquement, de leur suprématie sociale. Les villes de province ne sont pas en reste. Toutes se dotent d'une ou plusieurs promenades. On en compte par exemple trois à Sarragosse (El Coso, Santa Engracia, Torrero).

A la même époque apparaissent des préoccupations pour la sécurité publique et l'hygiène. Madrid, Barcelone, Valence se dotent des premières rues éclairées par des réverbères. En 1761, une pragmatique du Roi Charles III prévoit le pavage des rues et la pose de conduits d'évacuation des eaux usées. Le même texte interdit la libre circulation des porcs sur la voie publique, ce qui donne une idée de la situation dans laquelle se trouvait la capitale. Cependant, ces réformes décidées par le gouvernement ne furent pas très populaires, ce qui se comprend facilement lorsque l'on sait que les travaux furent imposés par le ministre Squilacce aux madrilènes et que ceux-ci furent

${ }^{9}$ Jacques Soubeyroux, Paupérisme..., p. 34, ainsi qu'un article plus récent, du même auteur, "Le Madrid de Charles III: un lieu de mémoire revisité par l'histoire», La ville et le monde ibérique et ibéro-américain, Poitiers, 1995, pp. 77-87.

${ }^{10}$ Jacques Soubeyroux, art. cit., p. 81. 
contraints de les payer de leurs deniers ${ }^{11}$. La «modernité» de ces réalisations doit donc être modulée: il semble que les réformes étaient plus inspirées par une préoccupation pour le prestige que par un souci pratique. D'ailleurs, il est révélateur que la ville espagnole du XVIIIe reste encore confinée dans ses anciennes limites, en particulier Madrid, ce qui n'était pas la marque d'un urbanisme tourné vers l'avenir. Ce n'est qu'au XIXe qu'apparaîtront les ensanches, extensions de l'enceinte urbaine hors des vieux murs, permettant d'augmenter la population par une croissance horizontale et non plus seulement verticale.

En parallèle à ces modifications architecturales, encore perceptibles de nos jours, une série de réformes va remodeler l'espace urbain dans son organisation administrative.

\subsection{Une administration dans des termes nouveaux}

La ville espagnole va donc connaître des changements dans la gestion de son espace administratif. D'abord, on voit apparaître de façon quasi systématique des plans de ville, chose qui jusqu'alors n'allait pas de soi. Séville, par exemple, qui fut la plate-forme du commerce colonial pendant près de deux siècles, ne posséda de plan digne de ce nom qu'en 1771. L'intendant Pablo de Olavide, à l'origine de cette réalisation, fit aussi numéroter les rues la même année et divisa l'espace en cuarteles de barrios. Les cuarteles étaient subdivisés en barrios, avec à leur tête l'alcalde de barrio, chargé de la surveillance de la circonscription. Cette division, qui avait été adoptée à Madrid en 1768 - huit cuarteles et huit barrios par cuartel_, fut aussi imposée à Barcelone l'année suivante - cinq cuarteles et huit barrios par cuartel. Dans la capitale, les alcaldes de cuarteles étaient nommés par le pouvoir. Les alcaldes de barrios, issus le plus souvent de la classe moyenne, étaient élus par les habitants de chaque quartier. Leur mission de police était évidente. Ils devaient dresser une liste de tous les habitants, comprenant l'adresse exacte ainsi que la profession, l'état, le nombre d'enfants et de domestiques de la famille. Ils devaient, par ailleurs, surveiller les tavernes, les tripots, ainsi que les auberges ou hôtels, afin d'en connaître et d'en répertorier tous les clients. Cette nouvelle organisation avait pour objet principal de rationaliser la surveillance de l'espace urbain. Elle faisait suite aux importantes

${ }^{11}$ Francisco J. Marín Perellón, «Madrid, ¿una ciudad para un rey?», Carlos III, Madrid y la Ilustración, Madrid, Siglo XXI, pp. 125-149. 
émeutes de 1766 (motín de Esquilache), qui avaient créé une véritable crainte chez les gouvernants.

Voilà donc, esquissées à grands traits, les dimensions de l'espace urbain dans l'Espagne des Lumières: une population plus dense et plus nombreuse, grâce à un solde migratoire positif, un espace urbain qui tend, timidement encore, à s'étendre vers la périphérie, mais qui plus généralement reste limité à la vieille enceinte, une architecture où l'on sent la volonté de créer, à travers les différentes réalisations, une image positive, une réorganisation administrative pour tenter de contrôler les mouvements populaires, potentiellement subversifs. Cette analyse, basée sur la masse d'archives laissée à l'Histoire (monuments, cartes, recensements, textes de loi) ne rend pas compte cependant de la dimension vécue de l'espace urbain.

\section{L'espace urbain dans les textes économiques}

Les économistes de l'époque furent sensibles au phénomène de mutation urbaine qui était en train de s'accomplir. Quelques analyses reconnaissent l'utilité de la ville dans le développement des connaissances et des sciences que l'on pourra ensuite appliquer à l'économie, mais elles sont peu nombreuses ${ }^{12}$. Ce qui domine, c'est l'image négative de l'espace urbain. Cette ville, qui fut l'objet de tant d'attentions, est très souvent critiquée. Bien qu'un nouvel espace urbain soit en train d'apparaître, comme en témoignent les réalisations architecturales et les réformes administratives, les pratiques économiques et le rapport ville-campagne restent en gros les mêmes qu'au siècle précédent.

\subsection{L'espace urbain, entrave économique}

D'abord, la ville était une véritable forteresse de privilèges. Elle exerçait sur les campagnes alentour une pression économique qui n'était pas toujours stimulante. Si l'existence d'un port dynamisait sans aucun doute l'agriculture de l'hinterland, les différentes législations

12Voir par exemple le prospectus du Semanario de Agricultura y Artes, Valladolid, Ámbito, 1997, p. 74; ainsi que Conde de Cabarrús, Cartas sobre los obstáculos que la naturaleza, la opinión y las leyes oponen a la felicidad pública escritas por el Conde de Cabarrús al señor don Gaspar de Jovellanos y precedidas de otra al Príncipe de la Paz, [1792], Madrid, Castellote, 1973, p. 155. 
autorisant les embargos sur les récoltes, interdisant la vente libre hors de l'espace urbain ou bloquant le prix des produits de première nécessité vendus sur le marché urbains, étaient autant d'entraves au développement économique rural ${ }^{13}$. Si la plupart des agglomérations conservent leur mur d'enceinte et leurs portes, c'est uniquement pour des raisons fiscales et commerciales. C'est, d'après Jovellanos, une des causes de la pénurie de certains produits car:

«Le laboureur, ne trouve aucun intérêt à les [ces produits] vendre à un prix arbitraire. Il est écarté des marchés par les formalités et les vexations qu'il subit et préfère ne pas les cultiver ${ }^{14}$ )

La solution proposée est simple, elle consiste en une libéralisation complète du marché urbain. Jovellanos, dans son projet de loi agraire, veut réformer la ville d'ancien régime et supprimer ses privilèges. Il veut la transformer en un espace homogène, indifférencié économiquement par rapport aux campagnes. Elle ne serait plus alors le lieu privilégié de l'échange, du marché, mais un marché possible, comme les autres. Ces propositions, inspirées de la doctrine libérale, apparaissaient déjà sous la plume de son ami et contemporain Cabarrús ${ }^{15}$.

Le deuxième point de la critique porte sur la concentration des richesses et des moyens par la ville. Un phénomène qui produit un effet négatif sur l'économie du pays. Ces analyses laissent entrevoir la décapitalisation de la campagne par la ville. Les grandes agglomérations étant le lieu de résidence des gros propriétaires (noblesse et clergé), la majorité des revenus agricoles circulaient en ville, au détriment de l'économie rurale, enfermée ainsi dans le sousdéveloppement. Cabarrús dans ses Cartas sobre los obstáculos que la Naturaleza, la opinión y las leyes oponen a la felicidad pública (Lettres sur les obstacles que la Nature, l'opinion et les lois opposent au bonheur public) analyse ainsi la situation:

${ }^{13}$ Marc Marti, Op. cit., chapitres 2 et 4.

14Jovellanos, Gaspar Melchor de, Espectáculos y diversiones públicas. Informe sobre la Ley Agraria, [1794], Madrid, Cátedra, 1986, p. 247-248: «El labrador, no hallando interés en venderlos a un precio arbitrario, y alejado de los mercados por las formalidades y las vejaciones que encuentra en ellos, toma el partido de no cultivarlos».

${ }^{15}$ Cabarrús, Op. cit., p. 154 sq. 
«Les liquidités ainsi que les propriétés se concentrent dans peu de villes entre les mains de quelques-uns, comment pourrait-il exister une circulation intérieure [...] Les tributs, les grands propriétaires, la daterie, les rentes ecclésiastiques, les tribunaux, les formidables administrations attirent à Madrid et dans quatre ou cinq grandes villes presque toute la substance du royaume $[\ldots]^{16}$,

Jovellanos propose une analyse quasi identique. Il fait aussi remarquer que ces capitaux, concentrés dans l'espace urbain, ne sont pas toujours utilisés à bon escient. Selon lui, certaines réalisations architecturales sont des constructions «inutiles» du point de vue économique:

«Il n'existe aucune nation qui n'aspire à établir sa splendeur sur la magnificence de ce que l'on appelle les monuments publics, et qui par conséquent n'ait rempli sa capitale, ses grandes et même ses petites villes de superbes édifices, et, alors que l'on manque de fonds pour les travaux nécessaires et profitables, il n'y en a pas une qui ne dépense avec prodigalité pour édifier des monuments purement ostentatoires, et qui plus est, pour s'en enorgueillir ${ }^{17}$ ».

Ce jugement semble d'abord moral, car il s'appuie sur une critique de l'orgueil («superbe, splendeur, magnificence, purement ostentatoire, s'enorgueillir»), mais la dimension économique n'est jamais perdue de vue «on manque de fonds pour les travaux nécessaires et profitables» (sous entendu des ponts, des routes et des ports). Cette analyse de la nocivité des réalisations architecturales urbaines est ensuite utilisée dans une comparaison ville/campagne:

«[...] il ne peut y avoir de contraste plus honteux que de voir les grandes capitales pleines de magnifiques portes, de places, de théâtres,

${ }^{16}$ Cabarrús, Ibid., "Reconcentrados así los signos como las propiedades en pocas ciudades, ¿cómo ha de haber circulación interior? [...] Los tributos, los grandes propietarios, la dataría, las encomiendas, los tribunales, las formidables oficinas, las pretensiones atraen a Madrid y a cuatro o cinco ciudades casi toda la sustancia del reino».

17Jovellanos, Op. cit., p. 321: «No hay nación que no aspire a establecer su esplendor sobre la magnificiencia de las que llaman obras públicas, que en consecuencia no haya llenado su corte, sus capitales y aun sus pequeñas ciudades y villas de soberbios edificios, y que mientras escasean sus fondos a las obras recomendadas por la necesidad y el provecho, no los derrame pródigamente para levantar monumentos de mera ostentación, y lo que es más, para envanecerse con ellos». 
de promenades et autres monuments ostentatoires, alors que, par manque de ports, de canaux et de routes, leur territoire est désertique et inculte, les villages qui en dépendent sont dépeuplés et remplis d'immondices et leurs habitants pauvres et misérables ${ }^{18}$ ».

L'aspect moral est toujours pris en compte («un contraste honteux»), mais la description insiste sur la différence entre la ville et la campagne, mettant en relief l'aspect misérable de cette dernière.

On arrive ainsi à la troisième critique, la plus fréquente dans les œuvres économiques de l'époque: l'espace urbain se peuple au détriment de la campagne, dont il absorbe les forces vives. Cabarrús insiste sur la disparition d'une partie des activités rurales industrielles, qui se sont réimplantées en ville, mais dont le prix de revient a augmenté. Il souligne, par ailleurs, que le développement d'activités manufacturières urbaines a privé l'agriculture d'une partie de sa main d'œuvre ${ }^{19}$. Pablo de Olavide fait une analyse quasi similaire:

«Les hommes que la nature a destinés aux travaux des champs ou aux travaux manuels abandonnent communément les villages où ils sont nés et où ils pourraient être très utiles. Ils s'en vont vers les villes les plus peuplées [...] et l'on peut craindre qu'ils y perdront leur innocence avant d'y avoir trouvé un travail ${ }^{20}$ ».

Mais chez lui, la critique de la concentration urbaine est aussi porteuse d'un discours social et moral conservateur. Les gens des campagnes naissent agriculteurs et la corruption se trouve en ville. C'est justement cette dimension morale qui sera souvent exploitée pour défendre l'idée d'une modification de la répartition spatiale de la population.

\subsection{La solution rêvée: la ville à la campagne}

18Ibid., pp. 321-322: «[...] no puede haber un contraste más vergonzoso que ver las grandes capitales llenas de magníficas puertas, plazas teatros, paseos y otros monumentos de ostentación, mientras por falta de puertos, canales y caminos está despoblado y sin cultivo su territorio, yermos y llenos de inmundicias sus pequeños lugares, y pobres y desnudos sus moradores».

${ }^{19}$ Cabarrús, Op. cit., p. 151.

${ }^{20}$ Pablo de Olavide, Cartas de Mariano a Antonio, El programa ilustrado de "El evangelio en triunfo", Aix en Provence, Service des publications, 1988, carta I: «Los hombres que la naturaleza destinó al campo o a los trabajos de las artes abandonan por lo común los lugares en donde nacieron y en que pudieran ser muy útiles. Se transportan a las ciudades populosas [...] y es muy de recelar que perderán su inocencia antes de encontrar un destino ». 
Déjà Campomanes, en 1774, pensait que «le véritable intérêt de l'État consiste à maintenir l'activité industrielle dispersée dans les villages et les hameaux ${ }^{21}$ ». Par «industria», on entend à l'époque toute activité de type manuel sans rapport direct avec l'agriculture. Dans l'économie de l'Espagne des Lumières, il peut s'agir tout aussi bien d'une production domestique que d'une manufacture. La réflexion que nous venons de citer démontre que c'est surtout la première que l'on entend développer. Campomanes considérait en effet que les premières concentrations manufacturières, qui étaient en train d'apparaître autour de Barcelone, étaient des plus nocives:

«En Catalogne, il faut développer les villages, en y transférant une grande partie de l'industrie qui s'est déplacée vers les villes, au détriment des villages et des campagnes ${ }^{22}$ ».

Ce désir de transférer une partie des villes à la campagne apparaît dans de nombreuses réflexions de l'époque. Pour Jovellanos, "une immense population rustique, répartie dans les campagnes, est pour l'État non seulement la promesse d'un peuple riche et laborieux mais aussi simple et vertueux ${ }^{23}$. . Le discours économique se double ainsi d'un discours moral. La ville apparaît comme un lieu de perdition, alors que la campagne se pare de toutes les vertus. Le Semanario de Agricultura y Artes dirigido a los párrocos (Hebdomadaire de l'Agriculture et des Arts destiné aux prêtres), dans son prospectus publicitaire, développe ce stéréotype moral et littéraire de façon complète:

«L'agriculture adoucit les mœurs. Elle produit des hommes simples, fidèles et honnêtes, qui se consacrent entièrement à elle et qui ne se laissent pas séduire par l'hypocrisie et la corruption des grandes

${ }^{21}$ Campomanes, Discurso sobre el fomento de la industria popular, [1774], Madrid, Ministerio de Hacienda, 1975, p. 76: «el verdadero interés del estado consiste en mantener dispersa la industria en caseríos y lugares chicos».

${ }^{22}$ Campomanes, Ibid., «En Cataluña conviene fomentar las aldeas, trasladando a ellas mucha parte de la industria que se va a las ciudades, en perjuicio de las aldeas y de los campos».

23Jovellanos, Informe, p. 183: «Sí, Señor: una inmensa población rústica derramada sobre los campos, no sólo promete al Estado un pueblo laborioso y rico, sino también sencillo y virtuoso». 
agglomérations. C'est ainsi que le cultivateur, préservé de la ville et de ses habitants, où qu'il habite sur la terre, est ingénu, doux et paisible ${ }^{24}$ ».

L'emploi quasi systématique de ce lieu commun moral dans le discours économique, décliné dans toutes ses variantes et possibilités, a de quoi surprendre. Nous pouvons cependant avancer quelques explications, qui pourraient tenir lieu de conclusion à cette communication.

\section{Conclusion: vécu psychologique et projections idéologiques}

Si nous résumons l'analyse, les principales critiques de l'espace urbain portent sur quatre points: les privilèges économiques, la concentration des richesses, l'attrait exercé sur la main d'œuvre des campagnes environnantes et la perdition morale qui s'ensuit pour les pauvres paysans venus s'installer à la ville. Ces quatre arguments concentrent la complexité de l'idéologie de l'élite dirigeante de cette époque.

L'attaque contre les privilèges économiques se fait à partir d'une idéologie d'inspiration clairement libérale, en particulier chez des auteurs comme Cabarrús et Jovellanos. L'analyse démontre la modernité de leur pensée, fondée sur la prise en compte des mécanismes du marché. Mais il y a peut-être un degré d'abstraction trop important (volontaire ou non). En effet, les privilèges de la ville étaient en fait destinés à protéger ses habitants les plus modestes des fortes hausses de prix des denrées de subsistance. La taxation était avant tout une mesure politique destinée à éviter les risques d'émeutes. La solution prônée par ces premiers libéraux était viable, à condition que la production agricole fût régulière, ce qui était loin d'être le cas. Et toutes les réformes économiques menées à ce moment là essaieront toujours de maintenir un difficile équilibre entre le libéralisme économique et la politique providentialiste propre de l'ancien Régime.

La critique de la concentration des richesses appartient à un registre différent. En effet, il est vrai que l'espace urbain était le lieu de

24Semanario, p. 73: «La agricultura suaviza las costumbres y hace a los hombres sencillos, fieles y honrados cuando, entregados enteramente a ella, no se dejan seducir por el trato engañoso y corrompido de los pueblos grandes. Y así es que el cultivador separado de ellos, de los que les frecuentan, en cualquier parte de la tierra que habite, es ingenuo, dulce y apacible». 
résidence des puissants propriétaires fonciers. Cependant, les critiques portent avant tout sur une inégalité spatiale, qui ne faisait en fait que retranscrire une flagrante inégalité sociale. C'est la notion d'espace urbain qui est attaquée, non l'origine de la fortune de ses résidents. Il est révélateur que les solutions envisagées soient toutes spatiales et non sociales. Jovellanos, Cabarrús, Olavide pensent résoudre la misère des campagnes en incitant les grands propriétaires à venir s'y installer, non en changeant le mode de production.

Enfin, la double condamnation -économique et morale- de l'immigration rurale révèle les intérêts et les craintes de l'élite sociale. L'exode des paysans, du point de vue économique, était un inconvénient pour les propriétaires fonciers. Elle se traduisait en effet par une hausse quasi-mécanique du coût de la main d'œuvre à cause de la réduction de l'offre sur le marché du travail, un phénomène qui fut sensible, par exemple, dans les campagnes catalanes. Ensuite, et là nous entrons dans des considérations psychologiques, qui avaient sans nul doute une importance non négligeable depuis les émeutes de 1766, les concentrations de nouveaux venus, surtout dans la capitale, étaient toujours suspectes. On craignait la subversion, car «une multitude inconnue est toujours prompte à se laisser séduire et entraîner par une sédition ${ }^{25 \eta}$.

Les économistes ne donnent donc qu'une vision partielle et partiale de la ville, en général assez négative, malgré quelques concessions. A notre avis, les textes retranscriraient un phénomène de croissance urbaine vécue négativement, ressentie comme dangereuse et traumatisante. Une image négative où, sans nul doute, l'expérience madrilène de la plupart des économistes a joué un rôle important. De plus, il nous semble que cette image de l'espace est déformée par des préjugés idéologiques qui tendent à occulter la réalité telle qu'on pouvait la lire dans les limites de l'espace urbain, où l'opulence n'était pas le fruit d'une mauvaise organisation spatiale mais bien le reflet des inégalités sociales et économiques.

${ }^{25}$ Cabarrús, Op. cit., p. 161: «[...] la reunión de una multitud desconocida, siempre pronta a ser arrastrada y seducida por cualquier sedición». Dans ce passage, Cabarrús critiquait l'établissement à Madrid d'une fabrique de salpêtre destinée à fabriquer de la poudre à canon. 


\section{Bibliographie}

Cabarrús, Conde de, Cartas sobre los obstáculos que la naturaleza, la opinión y las leyes oponen a la felicidad pública escritas por el Conde de Cabarrús al señor don Gaspar de Jovellanos y precedidas de otra al Príncipe de la Paz, [1792], Madrid, Castellote, 1973.

El Semanario de Agricultura y Artes dirigido a los Párrocos (17971808), selección e introducción por Elisabel Larriba y Gérard Dufour, Valladolid, Ámbito, 1997.

Equipo Madrid de Estudios Históricos, Carlos III, Madrid y la Ilustración, contradicciones de un proyecto reformista, Madrid, Siglo XXI, 1988.

Ferras, Robert, «"L'espace vécu": une direction de recherche en géographie», Imprévue n², Montpellier, CERS, 1982, pp. 17-27.

Jovellanos, Gaspar Melchor de, Espectáculos y diversiones públicas. Informe sobre la Ley Agraria, [1794], Madrid, Cátedra, 1986.

Marín Perellón, Francisco J., «Madrid, ¿una ciudad para un rey?», Carlos III, Madrid y la Ilustración, Madrid, Siglo XXI, 1988, pp. 125-149.

Marti, Marc, Ville et campagne dans l'Espagne des Lumières (17461808), Saint Étienne, Publications de l'Université de Saint Étienne, Cahiers du GRIAS, 1997.

Olavide, Pablo de, Cartas de Mariano a Antonio, El programa ilustrado de "El evangelio en triunfo", introduction de Gérard Dufour, Aix en Provence, Université de Provence, Service des publications, 1988.

Pérez Moreda, Vicente, Las crisis de mortalidad en la España interior. Siglos XVI-XIX, Madrid, 1981.

Rodríguez Pedro, Conde de Campomanes, Discurso sobre el fomento de la industria popular; Discurso sobre la educación popular de los artesanos y su fomento, [1774], ed. de Jhon Reeder, Madrid, 
Clásicos del pensamiento Económico Español, Instituto de Estudios Fiscales, Ministerio de Hacienda, 1975.

Soubeyroux, Jacques, Paupérisme et rapports sociaux à Madrid au XVIIIe siècle, Lille, atelier de reproduction des thèses universitaires de Lille III, 1978

Soubeyroux, Jacques, «Le Madrid de Charles III: un lieu de mémoire revisité par l'histoire», La ville et le monde ibérique et ibéroaméricain, Poitiers, 1995, pp. 77-87.

Vilar, Pierre, La Catalogne dans l'Espagne Moderne, SEVPEN, 1962.

Villes et Campagnes $X V$-XXe siècle, Centre d'Histoire économique et sociale de la région lyonnaise, Lyon, Presses Universitaires, 1977. 